H. Kazama, D. K. Kim and C. Y. Oh

Nagoya Math. J.

Vol. 157 (2000), 47-57

\title{
SOME REMARKS ON COMPLEX LIE GROUPS
}

\author{
H. KAZAMA, D. K. KIM AND C. Y. OH
}

\begin{abstract}
First we show that any complex Lie group is complete Kähler. Moreover we obtain a plurisubharmonic exhaustion function on a complex Lie group as follows. Let $\mathfrak{k}$ the real Lie algebra of a maximal compact real Lie subgroup $K$ of a complex Lie group $G$. Put $q:=\operatorname{dim}_{\mathbb{C}} \mathfrak{k} \cap \sqrt{-1} \mathfrak{k}$. Then we obtain that there exists a plurisubharmonic, strongly $(q+1)$-pseudoconvex in the sense of Andreotti-Grauert and $K$-invariant exhaustion function on $G$.
\end{abstract}

\section{$\S 1$. Introduction}

To get our aim of this paper, we may assume that every complex Lie group is always connected throughout this paper.

Let $G$ be a complex Lie group of complex dimension $n$ and $\mathfrak{k}$ the real Lie algebra of a maximal compact real Lie subgroup $K$ of $G$. Put $q:=$ $\operatorname{dim}_{\mathbb{C}} \mathfrak{k} \cap \sqrt{-1} \mathfrak{k}$. Since all maximal compact subgroups are conjugate each other([2]), the integer $q$ is independent of a choice of a maximal compact subgroup. Concerning the pseudoconvexity of complex Lie groups, we have the following theorem which was proved in [10] and, partially, in $[3,4]$.

TheOREm 1. There exists a $C^{\infty}$ plurisubharmonic function $\varphi$ on $G$ satisfying (1) and (2).

(1) The Levi form of $\varphi$ is positive semidefinite and has $n-q$ positive eigenvalues at every point of $G$, in other words, $\varphi$ is plurisubharmonic and strongly $(q+1)$-pseudoconvex on $G$ in the sense of [1].

(2) $\varphi$ is an exhaustion function on $G$, i.e., for any $c \in \mathbb{R}$.

$$
\{x \in G \mid \varphi(x)<c\} \subset \subset G .
$$

On the Stein group $G L(n, \mathbb{C})$ there exists a natural strongly plurisubharmonic exhaustion function

Received September 21, 1998. 


$$
\Phi(a):=\operatorname{trace}\left(a a^{*}\right)+\frac{1}{\operatorname{det}\left(a a^{*}\right)}
$$

$(a \in G L(n, \mathbb{C}))$. This function $\Phi$ is invariant with respect to the left and right actions of the unitary subgroup $U(n):=\left\{a \mid a \in G L(n, \mathbb{C}), a^{-1}=\right.$ $\left.a^{*}\right\}$ which is a maximal compact real Lie subgroup of $G L(n, \mathbb{C})$, i.e., for $a \in G L(n, \mathbb{C})$ and $x, y \in U(n)$,

$$
\Phi(x a y)=\Phi(a) .
$$

The purpose of this paper is to consider whether or not there exists a plurisubharmonic and strongly $(q+1)$-pseudoconvex exhaustion function in the sense of [1] on $G$ which is invariant on a given maximal compact real Lie subgroup $K$ and to show, as it's application, the existence of complete Kähler metric for every complex Lie group.

We wish to thank Prof. Hideki Kosaki and Prof. Mitsuo Morimoto who gave important advice for us to prove Lemma 3 and Theorem 3, respectively.

\section{$\S 2$. Linear groups and abelian Lie groups}

A closed complex Lie subgroup of the complex general linear group $G L(k, \mathbb{C})$ for some positive integer $k$ is said to be a closed complex linear group.

LEMma 1. Let $G$ be a closed complex linear group and $K$ a maximal compact subgroup of $G$. Then there exists a $C^{\infty}$ function

$$
\varphi: G \longrightarrow \mathbb{R}
$$

such that

(1) $\varphi$ is strongly plurisubharmonic,

(2) $\varphi$ is an exhaustion function on $G$, i.e., for any $c \in \mathbb{R}$.

$$
\{x \in G \mid \varphi(x)<c\} \subset \subset G,
$$

(3) $\varphi$ is $K$-invariant, that is,

$$
\varphi(x)=\varphi(y x z)
$$

for any $x \in G, y, z \in K$. 
Proof. Let $G$ be a closed complex Lie subgroup of the complex general linear group $G L(k, \mathbb{C})$. There exists a maximal compact subgroup $K_{1}$ of $G L(k, \mathbb{C})$ such that

$$
K \subset K_{1}
$$

Since all maximal compact subgroups are conjugate each other $([2])$, one can find $a \in G L(k, \mathbb{C})$ so that

$$
a K_{1} a^{-1}=U(k)
$$

where $U(k)$ is the unitary subgroup of $G L(k, \mathbb{C})$. Taking a function

$$
\varphi(x):=\Phi\left(a x a^{-1}\right)
$$

where $\Phi(x)=\operatorname{trace}\left(x x^{*}\right)+\frac{1}{\operatorname{det}\left(x x^{*}\right)}$, we get the assertion of this lemma.

In the case of complex abelian Lie groups we obtain a similar result as Lemma 1.

LEMMA 2. Let $G$ be a complex abelian Lie group of complex dimension $n$ and $K$ a maximal compact subgroup of $G$. Then there exists a $K$-invariant $C^{\infty}$ function

$$
\varphi: G \longrightarrow \mathbb{R}
$$

satisfying the same statements (1) and (2) in Theorem 1.

Proof. Let $e$ be the unit element of the complex abelian Lie group $G$. Put

$$
G^{0}:=\{x \mid f(x)=f(e) \text { for every holomorphic function } f \text { on } G\}
$$

From the result of [7] $G^{0}$ is a complex Lie subgroup of $G$ which is a toroidal group, that is, a connected complex Lie group without nonconstant holomorphic functions and there exists a lattice $\Gamma$ of $\mathbb{C}^{m}$ such that $G^{0} \cong \mathbb{C}^{m} / \Gamma$, where $m$ is the complex dimension of $G^{0}$. We may assume $\Gamma=\mathbb{Z}\left\{e_{1}, e_{2}, \ldots, e_{m}, v_{1}, v_{2}, \ldots, v_{q_{0}}\right\}$, where $e_{i}$ is the $i$-th standard unit vector of $\mathbb{C}^{m}$ and $e_{1}, e_{2}, \ldots, e_{m}, v_{1}, v_{2}, \ldots, v_{q_{0}}$ are $\mathbb{R}$-linearly independent, and $K^{0} \cong \mathbb{R}\left\{e_{1}, e_{2}, \ldots, e_{m}, v_{1}, v_{2}, \ldots, v_{q_{0}}\right\} / \Gamma$. Let $v_{i}=\left(v_{i 1}, v_{i 2}, \ldots, v_{i m}\right)$ and $v_{i}=\operatorname{Re} v_{i}+\sqrt{-1} \operatorname{Im} v_{i}\left(\operatorname{Re} v_{i}, \operatorname{Im} v_{i} \in \mathbb{R}^{m}\right)$. Since $e_{1}, e_{2}, \ldots, e_{m}, v_{1}, v_{2}, \ldots, v_{q_{0}}$ are $\mathbb{R}$-linearly independent, $\operatorname{Im} v_{1}, \ldots, \operatorname{Im} v_{q_{0}}$ are $\mathbb{R}$-linearly independent. Without loss of generality we may assume the $q_{0} \times q_{0}$ real matrix 
$\left(v_{i j} ; 1 \leq i, j \leq q_{0}\right)$ is invertible. We put $v_{q_{0}+1}:=\sqrt{-1} e_{q_{0}+1}, v_{q_{0}+2}$ $:=\sqrt{-1} e_{q_{0}+2}, \ldots, v_{m}:=\sqrt{-1} e_{m}$. Then $e_{1}, e_{2}, \ldots, e_{m}, v_{1}, v_{2}, \ldots, v_{q_{0}}$, $v_{q_{0}+1}, \ldots, v_{m}$ are $\mathbb{R}$-linearly independent vectors of $\mathbb{C}^{m}$. For any $z=$ $\left(z_{1}, z_{2}, \ldots, z_{m}\right)$ there exists a unique vector $\left(t_{1}, t_{2}, \ldots, t_{2 m}\right) \in \mathbb{R}^{2 m}$ such that

$$
z=\sum_{i=1}^{m} t_{i} e_{i}+\sum_{i=1}^{q_{0}} t_{m+i} \sqrt{-1} \operatorname{Im} v_{i}+\sum_{i=q_{0}+1}^{m} t_{m+i} v_{i} .
$$

We define the exhaustion function

$$
\psi: \mathbb{C}^{m} / \Gamma \ni z+\Gamma \longmapsto \sum_{i=q+1}^{m} t_{m+i}^{2} \in \mathbb{R}
$$

on $\mathbb{C}^{m} / \Gamma$. We denote by $A=\left(a_{i j}\right)$ the inverse matrix of

$$
\left(\begin{array}{c}
\operatorname{Im} v_{1} \\
\cdot \\
\cdot \\
\operatorname{Im} v_{q_{0}} \\
e_{q_{0}+1} \\
\cdot \\
\cdot \\
e_{m}
\end{array}\right)
$$

Let $x_{i}:=\operatorname{Re} z_{i}$ and $y_{i}:=\operatorname{Im} z_{i}$. Then we have $t_{m+i}=\sum_{k=1}^{m} y_{k} a_{k i}(i \geq$ $\left.q_{0}+1\right)$. The Levi form of $\psi$ is given by

$$
\left(\frac{\partial^{2} \psi(z+\Gamma)}{\partial z_{i} \partial \bar{z}_{j}}\right)=\frac{1}{4}\left(\frac{\partial^{2} \psi}{\partial y_{i} \partial y_{j}}\right)=\frac{1}{2} B B^{t}
$$

where $B$ is the matrix

$$
\left(\begin{array}{cccccc}
0 & \ldots & 0 & a_{1} q_{0}+1 & \ldots & a_{1 m} \\
0 & & 0 & a_{2} q_{0}+1 & \ldots & a_{2 m} \\
\vdots & \ddots & \vdots & \vdots & \ddots & \vdots \\
0 & \ldots & 0 & a_{m} q_{0}+1 & \ldots & a_{m m}
\end{array}\right)
$$


Since $B$ is a real $(m, m)$-matrix of rank $m-q_{0}, \frac{1}{2} B B^{t}$ is positive semidefinite with $m-q_{0}$ positive eigenvalues. By the definition of $\psi$ we can see that $\psi\left(z+z^{*}+\Gamma\right)=\psi(z+\Gamma)$ for any $z \in \mathbb{C}^{m}$ and $z^{*} \in K^{0}$. This means $\psi$ is $K^{0}$-invariant. By the result of [8] $G \cong G^{0} \times \mathbb{C}^{* p} \times \mathbb{C}^{r}$ for some non-negative integers $p$ and $r$ with $m+p+r=n$. Then we may assume $G=G^{0} \times \mathbb{C}^{* p} \times \mathbb{C}^{r}$. We take the function

$$
\varphi(z+\Gamma, \xi, \eta):=\psi(z+\Gamma)+\sum_{i=1}^{p}\left(\frac{1}{\left|\xi_{i}\right|^{2}}+\left|\xi_{i}\right|^{2}\right)+\sum_{j=1}^{r}\left|\eta_{j}\right|^{2}
$$

for $(z+\Gamma, \xi, \eta) \in G^{0} \times \mathbb{C}^{* p} \times \mathbb{C}^{r}$, where $\xi=\left(\xi_{1}, \ldots, \xi_{p}\right) \in \mathbb{C}^{* p}$ and $\eta=$ $\left(\eta_{1}, \ldots, \eta_{r}\right) \in \mathbb{C}^{r}$. Since $G$ is abelian, $G$ has the unique maximal compact subgroup $K=K^{0} \times\left\{\xi ;\left|\xi_{i}\right|=1\right\} \times 0 \subset G^{0} \times \mathbb{C}^{* p} \times \mathbb{C}^{r}$. It is easy to show that $\varphi$ is $K$-invariant and the Levi form of $\varphi$ has $n-q_{0}$ positive eigenvalues at every point of $G$. Let $\mathfrak{k}$ be the Lie algebra of $K$. Then

$$
\mathfrak{k}=\mathbb{R}\left\{e_{1}, e_{2}, \ldots, e_{m}, v_{1}, v_{2}, \ldots, v_{q_{0}}\right\} \oplus \mathbb{R}^{q} \oplus 0
$$

that is a real Lie subalgebra of the Lie algebra $\mathbb{C}^{m} \times \mathbb{C}^{p} \times \mathbb{C}^{r}$ of $G$.

Hence $\mathfrak{k} \cap \sqrt{-1} \mathfrak{k}=\mathbb{R}\left\{e_{1}, e_{2}, \ldots, e_{m}, v_{1}, v_{2}, \ldots, v_{q_{0}}\right\} \cap$ $\mathbb{R}\left\{\sqrt{-1} e_{1}, \sqrt{-1} e_{2}, \ldots, \sqrt{-1} e_{m}, \sqrt{-1} v_{1}, \sqrt{-1} v_{2}, \ldots, \sqrt{-1} v_{q_{0}}\right\}=$ $\mathbb{C}\left\{\operatorname{Im} v_{1}, \ldots, \operatorname{Im} v_{q_{0}}\right\}$. Then we have

$$
q=\operatorname{dim}_{\mathbb{C}} \mathfrak{k} \cap \sqrt{-1 \mathfrak{k}}=q_{0}
$$

\section{$\S 3$. Complete Kähler metric on a complex Lie group}

Prof. Hiroshi Yamaguchi proposed the following question.

QUESTION. Is any complex Lie group a Kähler manifold?

In this section we will give the affirmative answer to this question, that is, we will obtain the following theorem.

\section{TheOrem 2. Any complex Lie group is complete Kähler.}

Proof. Let $\mathfrak{g}$ be the Lie algebra of $G$ and $K_{C}$ the complex Lie subgroup with the Lie subalgebra $\mathfrak{k}_{C}:=\mathfrak{k}+\sqrt{-1} \mathfrak{k}$. Then $K_{C}$ is a closed complex Lie subgroup of $G([5])$. Put $a:=\operatorname{dim}_{C} G / K_{C}$. Then $G$ is biholomorphic onto $K_{C} \times \mathbb{C}^{a}([5])$. From this fact we may assume $G=K_{C}$. By the result of [5] there exist closed connected complex Lie subgroups $S$ and $Z$ such that 
(1) $S$ is semi-simple,

(2) $Z$ is the connected center of $G$,

(3)

$$
\rho: Z \times S \ni(x, y) \longmapsto x y \in G
$$

is a finite covering homomorphism.

$Z$ is a connected complex abelian Lie group and then is isomorphic onto $\mathbb{C}^{p} / \Gamma$ for some discrete subgroup $\Gamma$ of $\mathbb{C}^{p}$. Let $\left(z_{1}, \ldots, z_{p}\right)$ be the canonical coordinate system of $\mathbb{C}^{p}$. The complete Kähler metric

$$
\sum_{i=1}^{p} d z_{i} d \overline{z_{i}}
$$

induces a complete Kähler metric on $Z$ that is $\Gamma$-invariant. Since Ker $\rho$ is a finite subgroup of $Z \times S$, there exist maximal compact subgroups $K_{Z}$ and $K_{S}$ of $Z$ and $S$, respectively such that $\operatorname{Ker} \rho \subset K_{Z} \times K_{S}$. Since $S$ is semisimple, $S$ is isomorphic onto a complex linear group and further we may assume $S$ is a closed complex Lie subgroup of $G L(k, \mathbb{C})$ for some positive integer $k([6])$. By Lemma 1 there exists a $C^{\infty}$ strongly plurisubharmonic exhaustion function

$$
\varphi: S \longrightarrow \mathbb{R}
$$

that is $K_{S}$-invariant. The form

$$
\sum_{i=1}^{p} d z_{i} d \overline{z_{i}}+\sum_{j, \ell=1}^{s} \frac{\partial^{2} \varphi}{\partial w_{j} \partial \overline{w_{\ell}}} d w_{j} d \overline{w_{\ell}}
$$

is $\operatorname{Ker} \rho$-invariant and then induces a Kähler metric on $G \cong Z \times S / \operatorname{Ker} \rho$, where $s:=\operatorname{dim}_{\mathbb{C}} S$ and $\left(w_{1}, \ldots, w_{s}\right)$ is a local coordinate system of $S$. From the technique of Nakano (Proposition 1, [9]), we can find a strictly convex increasing $C^{\infty}$ function

$$
\chi:(0, \infty) \longrightarrow(0, \infty)
$$

such that the Kähler metric

$$
\sum_{i=1}^{p} d z_{i} d \overline{z_{i}}+\sum_{j, \ell=1}^{s} \frac{\partial^{2} \chi(\varphi)}{\partial w_{j} \partial \overline{w_{\ell}}} d w_{j} d \overline{w_{\ell}}
$$

is complete. 


\section{$\S 4$. Invariant plurisubharmonic exhaustion functions}

LEMMA 3. Let $K$ be a compact topological space with a positive measure $m$ and $a_{i j}(x): K \longrightarrow \mathbb{C}$ be continuous functions for $1 \leq i, j \leq n$. If $n \times n$ matrix

$$
A(x):=\left(a_{i j}(x)\right)
$$

is positive semidefinite Hermitian and has $n-q$ positive eigenvalues at any point $x$ of $K$, then

$$
B:=\left(\int_{K} a_{i j}(x) d m\right)
$$

is positive semidefinite and has at least $n-q$ positive eigen values.

Proof. We put $w:={ }^{t}\left(w_{1}, \ldots, w_{n}\right)$ for $\left(w_{1}, \ldots, w_{n}\right) \in \mathbb{C}^{n}$. Since

$$
(B w, w):=\sum_{i, j=1}^{n} w_{j} \int_{K} a_{i j}(x) d m \overline{w_{i}}=\int_{K} \sum_{i, j=1}^{n} w_{j} a_{i j}(x) \overline{w_{i}} d m \geq 0,
$$

the matrix $B$ is positive semidefinite Hermitian. Then there exists a unitary matrix $U$ such that $B={ }^{t} \bar{U} \Lambda U$, where

$$
\Lambda=\left(\begin{array}{ccccc}
\lambda_{1} & 0 & 0 & \ldots & 0 \\
0 & \lambda_{2} & \ddots & \ddots & 0 \\
0 & \ddots & \ddots & \ddots & \vdots \\
\vdots & \ddots & \ddots & \ddots & 0 \\
0 & \ldots & \ldots & 0 & \lambda_{n}
\end{array}\right)
$$

and $\lambda_{i}$ are non-negative eigenvalues of $B$. We get a positive semidefinite Hermitian matrix

$$
\sqrt{B}:={ }^{t} \bar{U}\left(\begin{array}{ccccc}
\sqrt{\lambda_{1}} & 0 & 0 & \ldots & 0 \\
0 & \sqrt{\lambda_{2}} & \ddots & \ddots & 0 \\
0 & \ddots & \ddots & \ddots & \vdots \\
\vdots & \ddots & \ddots & \ddots & 0 \\
0 & \ldots & \ldots & 0 & \sqrt{\lambda_{n}}
\end{array}\right) U .
$$

If $(B w, w)=0$, then $\left((\sqrt{B})^{2} w, w\right)=(\sqrt{B} w, \sqrt{B} w)=0$ and hence $\sqrt{B} w=0 . \sqrt{B} w=0$ implies $B w=(\sqrt{B})(\sqrt{B}) w=0$. This shows that

$$
B w=0
$$


if and only if

$$
(B w, w)=\sum_{i, j=1}^{n} w_{j} \int_{K} a_{i j}(x) d m \overline{w_{i}}=0 .
$$

We consider the eigen space

$$
E_{0}:=\{w \mid B w=0\}=\{w \mid(B w, w)=0\}
$$

of the eigenvalue 0 of $B$. Since

$$
(B w, w)=\int_{K} \sum_{i, j=1}^{n} w_{i} a_{i j}(x) \overline{w_{j}} d m=0
$$

we have $\sum_{i, j=1}^{n} w_{j} a_{i j}(x) \overline{w_{i}}=0$ for any $x \in K$. Then we obtain

$$
E_{0} \subset \cap_{x \in K}\{w \mid A(x) w=0\} \subset\left\{w \mid A\left(x_{0}\right) w=0\right\}
$$

for any point $x_{0} \in K$. From the assumption it follows that

$$
\operatorname{dim}_{C}\left\{w \mid A\left(x_{0}\right) w=0\right\} \leq q
$$

and then $\operatorname{dim}_{C} E_{0} \leq q$.

The following Theorem 3 implies Theorem 1. Moreover, using a different method from those given by [3], [4] and S. Takeuchi [10], we prove Theorem 3 here.

TheOREm 3. Let $G$ be a complex Lie group, $K$ a maximal compact subgroup of $G$. Put $q:=\operatorname{dim}_{\mathbb{C}} \mathfrak{k} \cap \sqrt{-1} \mathfrak{k}$. Then there exists a $C^{\infty}$ function

$$
\varphi: G \longrightarrow \mathbb{R}
$$

such that

(1) $\varphi$ is plurisubharmonic and strongly $(q+1)$-pseudoconvex on $G$ in the sense of $[1]$,

(2) $\varphi$ is an exhaustion function on $G$, i.e., for any $c \in \mathbb{R}$.

$$
\{z \in G \mid \varphi(z)<c\} \subset \subset G
$$


(3) $\varphi$ is K-invariant, that is,

$$
\varphi(z)=\varphi(a z b)
$$

for any $z \in G, a, b \in K$.

Proof. We obtain closed connected Lie subgroups $K_{C}, S, Z, K_{S}, K_{Z}$ and a finite covering homomorphism

$$
\rho: Z \times S \ni(x, y) \longmapsto x y \in K_{C}
$$

and $G$ is biholomorphic onto $K_{C} \times \mathbb{C}^{a}$ as in the proof of Theorem 2. Put $n:=\operatorname{dim} G, n_{1}:=\operatorname{dim} Z$ and $n_{2}:=\operatorname{dim} S\left(n=n_{1}+n_{2}+a\right)$. Since $Z$ is abelian, we have isomorphisms

$$
Z \cong G^{0} \times \mathbb{C}^{* p} \times \mathbb{C}^{r}
$$

and $G^{0} \cong \mathbb{C}^{m} / \Gamma\left(n_{1}=m+p+r\right)$, where we put $G^{0}:=\{x \mid f(x)=$ $f(e)$ for every holomorphic function $f$ on $Z\}$ and $\Gamma=\mathbb{Z}\left\{e_{1}, e_{2}, \ldots, e_{m}\right.$, $\left.v_{1}, v_{2}, \ldots, v_{q_{0}}\right\}$, using the notations in the proof of Lemma 2. By Lemmas 1 and 2 we obtain a strongly plurisubharmonic exhaustion function $\varphi_{S}$ : $S \longrightarrow \mathbb{R}$ and a plurisubharmonic exhaustion function $\varphi_{Z}: Z \longrightarrow \mathbb{R}$, where the Levi form of $\varphi_{Z}$ has $n_{1}-q_{0}$ positive eigen values at every point. Since $\varphi_{S}$ and $\varphi_{Z}$ are $K_{S^{-}}$and $K_{Z}$-invariant, respectively and $\operatorname{Ker} \rho \subset K_{Z} \times K_{S}$, they induce a plurisubharmonic exhaustion function

$$
\varphi_{K_{C}}:=\varphi_{Z}+\varphi_{S}: K_{C} \longrightarrow \mathbb{R}
$$

The Levi form of $\varphi_{K_{C}}$ has $n-a-q_{0}$ positive eigenvalues at every point. We take a strongly plurisubharmonic exhaustion function

$$
\varphi_{a}: \mathbb{C}^{a} \ni\left(w_{1}, \ldots, w_{a}\right) \mapsto \sum_{i=1}^{a}\left|w_{i}\right|^{2} \in \mathbb{R} .
$$

We put

$$
\varphi_{G}:=\varphi_{K_{C}}+\varphi_{a}: G \longrightarrow \mathbb{R} .
$$

Then $\varphi_{G}$ is a plurisubharmonic exhaustion function whose Levi form has $n-q_{0}$ positive eigenvalues at every points. Since the maximal compact 
subgroup $K$ is a Lie group, we can obtain positive left (or right) invariant Haar measure $\mu_{\ell}\left(\right.$ or $\mu_{r}$ ) on $K$, respectively. Finally we obtain a function

$$
\varphi(z):=\int_{x \in K} \int_{y \in K} \varphi_{G}(y z x) d \mu_{\ell}(x) d \mu_{r}(y) .
$$

Since it's Levi form is

$$
\left(\frac{\partial^{2} \varphi(z)}{\partial z_{i} \partial \overline{z_{j}}}\right)=\left(\int_{x \in K} \int_{y \in K} \frac{\partial^{2} \varphi_{G}(y z x)}{\partial z_{i} \partial \overline{z_{j}}} d \mu_{\ell}(x) d m u_{r}(y)\right),
$$

by Lemma 3 this is positive semi-definite and has at least $n-q_{0}$ positive eigenvalues at every point of $G$. Furthermore, for $a, b \in K$

$$
\begin{aligned}
\varphi(a z b) & =\int_{x \in K} \int_{y \in K} \varphi_{G}\left(\left(\begin{array}{ll}
y & a
\end{array}\right)\left(\begin{array}{l}
b \\
y
\end{array}\right)\right) d \mu_{\ell}(x) d \mu_{r}(y) \\
& =\int_{x \in K} \int_{y \in K} \varphi_{G}(y z x) d \mu_{\ell}(x) d \mu_{r}(y) \\
& =\varphi(z) .
\end{aligned}
$$

Let $\mathfrak{k}_{Z}$ and $\mathfrak{k}_{S}$ be the Lie algebras of $K_{Z}$ and $K_{S}$, respectively. There exists $a \in G$ such that $K_{S} \subset a U(k) a^{-1}$. The isomorphism $\alpha: G \ni x \mapsto a x a^{-1} \in G$ induces the isomorphism $\alpha: \mathfrak{g} \mapsto \mathfrak{g}$ of complex Lie algebras. Since the Lie algebra $\mathfrak{u}=\{x: \bar{t}+x=0\}$ of all skew-Hermitian matrices is the Lie algebra of the unitary group $U(k)$ and $\mathfrak{k}_{S} \cap \sqrt{-1} \mathfrak{k}_{S} \subset \alpha(\mathfrak{u} \cap \sqrt{-1} \mathfrak{u})$, we have $\mathfrak{u} \cap \sqrt{-1} \mathfrak{u}=0$ and then

$$
q=\operatorname{dim}_{\mathbb{C}} \mathfrak{k} \cap \sqrt{-1} \mathfrak{k}=\operatorname{dim}_{\mathbb{C}} \mathfrak{k}_{Z} \cap \sqrt{-1} \mathfrak{k}_{Z}=q_{0} .
$$

\section{REFERENCES}

[1] A. Andreotti and H. Grauert, Théorèmes de finitude pour la cohomologie des espaces complexes, Bull. Soc. Math. France, 99 (1962), 193-259.

[2] K. Iwasawa, On some types of topological groups, Annals of Math., 50 (1949), 507-558.

[3] H. Kazama, On pseudoconvexity of complex abelian Lie groups, J. Math. Soc. Japan, 25 (1973), 329-333.

[4] H. Kazama, On pseudoconvexity of complex Lie groups, Mem. Fac. Sci. Kyushu Univ., 27 (1973), 241-247.

[5] Y. Matsushima, Espaces homogènes de Stein des groupes de Lie complexes, Nagoya Math. J., 16 (1960), 205-218. 
[6] Y. Matsushima and A. Morimoto, Sur certains espaces fibrés holomorphes sur une variété de Stein, Bull. Soc. Math. France, 88 (1960), 137-155.

[7] A. Morimoto, Non-compact complex Lie groups without non-constant holomorphic functions, Proc. Conf. on Complex Analysis, Minneapolis (1965), 256-272.

[8] A. Morimoto, On the classification of non-compact complex abelian Lie groups, Trans. Amer. Math. Soc., 123 (1966), 200-228.

[9] S. Nakano, On the inverse of monoidal transformation, Publ.RIMS, 6 (1970), 483-502.

[10] S. Takeuchi, On completeness of holomorphic principal bundles, Nagoya Math. J., 57 (1974), 121-138.

H. Kazama

Graduate School of Mathematics

Kyushu University

Fukuoka, 810-8560

Japan

D. K. Kim

Department of Mathematics

Chonbuk National University

Chonju, Chonbuk 561-756

Korea

C. Y. Oh

Department of Applied Mathematics

Yosu National University

Yosu, Chollanam 550-749

Korea 